\title{
Un homme se change en homme: multiplication des travestissements dans Talons aiguilles de Pedro Almodovar
}

\author{
Olga Duhamel
}

\section{A Man Gets Changed into a Man: Cross-dressing Multiplied in Pedro Almodovar's High Heels}

In French, 'genre' means both gender and literary kind; this pair of meanings links the concept of gender to issues of convention, imitation, identity as the effect of a generic performance. Masculinity, however, is popularly conceived as natural, self-evident, without need of artifice; it is conceived as such in opposition to a femininity which is all about artifice and masquerade - according to orthodox psychoanalytic theory, a overcompensatory corporeal vanity symptomatic of penis envy in women. But perhaps this model of one-sided envy is rigged: the self-sufficient, integral masculine position certainly has the ring of sexual indifference, of a system in which all value is measured against a fantasmatic masculineneutral. What happens when one considers reciprocal envy as an inescapable condition of functionning within sexual difference? And when, furthermore, there is no automatic and natural adequation of biological sex and gender, and no gender which is independent of representation, performance, a practice of reproducing and reading marks on the body?

Pedro Almodovar's 1991 film, High Heels, challenges the selfevidence of the supposedly unmarked masculine gender through the character Letal-Dominguez-Hugo, a 'female impersonator' ('lethal' woman) who also cross-dresses in his two incarnations of the male-the potent judge, Dominguez, in fake beard and tie (mandatory mark of virility), and the 'sincere' guy, "ego-Hugo," in jeans and t-shirt. The true identity of this individual is readable neither in the eyes (those windows on the soul) nor in the 'evidence' of his biological sex: significantly, this emblem is insufficient as a marker of identity without an additional, accidental sign, a beauty spot which permits Letal-Dominguez-Hugo's lovers to piece together his multiple performances. 
«Le masculin l'emporte», tel était le cri de guerre des petits garçons, à l'école primaire, après une des premières classes sur l'accord du nom réglé par le masculin en français. Il est donc normal que l'on reste longtemps piégé par un fantasmatique masculin neutre. Le masculin serait générique, il serait neutre, plus que neutre, il serait naturel, il n'aurait pas besoin d'artifice, il se donnerait en une fois, il aurait ce que les deux sexes désirent, il serait évident et il l'emporterait même sur le féminin; ça vient de loin. Toutes ces idées viennent de loin et nous entraînent loin, jusqu'à faire glisser le sens du neutre, du ni l'un ni l'autre. Du neuter, nous passons à un implacable neutre cumulateur, à un neutre qui se veut à la fois et l'un (le masculin) et l'autre.

Boucles d'oreilles, petites chaussures ou talons aiguilles, rouge à lèvres, fards de toutes sortes, bas résilles, etc., seraient, selon le modèle freudien, les attributs féminins qui viendraient masquer le manque. Freud, c' est un vieux débat déjà, écrit ceci à propos des manifestations du. féminin:

L'effet de l'envie du pénis est encore impliqué dans la vanité corporelle de la femme, dans la mesure où elle doit estimer d'autant plus haut ses attraits en tant que dédommagement tardif de son infériorité sexuelle initiale. (177)

Du côté du masculin, du genre qui l'a, il n'y aurait pas de préoccupations vestimentaires aussi importantes, sinon celle de ne rien perdre, de ne pas montrer de brèche, de manque. L'homme, pour être homme, n'aurait qu'une préoccupation: ne pas perdre son sexe. Il ne serait pas envieux de la femme, il aurait l'angoisse d'être castré. Ce n'est pas l'avis du psychanalyste Felix Boehm, contemporain de Freud qui, dans un texte de 1930, «Le complexe de féminité chez l'homme», écrit:

Le refoulement du désir d'être une femme engendre chez beaucoup d'hommes une aversion contre tout ce qui est féminin.[...] Derrière la haine de toutes les particularités féminines des femmes, se cache le désir de posséder ces mêmes particularités, et en outre l'envie de posséder le pénis des femmes, supposé plus grand: puisque tu es si grande, tu dois avoir un fait-pipi comme un cheval dit le petit Hans à sa mère. (286-287)

Pour Boehm, l'homme serait lui aussi aux prises avec l'envie, ce qui est une exception pour la psychanalyse orthodoxe qui voit l'envie du côté des femmes uniquement. Pour cette psychanalyse lesexe mâle, et lui 
seul, est ce par rapport à quoi s'articulent les deux sexes. Étant châtrée, la femme doit donc dépasser cette envie du pénis qui constitue un stade obligé dans son développement; elle doit dépasser son envie pour atteindre un développement complet de son identité sexuelle et devenir une femme. Quant à l'homme, effrayé de découvrir qu'il y aurait des êtres dépourvus de sexe, il garderait sa vie durant l'angoisse de devenir un de ces êtres à qui on a sectionné le membre. Quelque chose coince. Le modèle nous piège. La question de l'envie est-elle à l'avance piégée?

En fait, les qualités du masculin et du féminin changent d'une époque à l'autre, d'un lieu à un autre, d'un individu à un autre, etc.; elles changent et se transforment. Mais il n'y a jamais un élément biologique décisif qui détermine entièrement le fait d'être un homme masculin. Ce qui demeure difficile, c'est de fonctionner dans la différence, une différence qui entraîne l'envie, de part et d'autre des deux sexes. Ce qui demeure difficile c'est d'accepter que le genre nécessite le marquage et qu'il n'existe que par les marques, que ce genre corresponde ou non au sexe biologique.

Nous tenterons ici de comprendre comment fonctionne le personnage de Letal-Dominguez-Hugo dans Talons aiguilles (1991), à la fois travesti en femme et en homme. Mais auparavant, rappelons les grandes lignes de l'intrigue tarabiscotée du film d'Almodovar. Becky, une chanteuse célèbre des années soixante, rentre à Madrid qu'elle a quitté depuis plus de quinze ans. Sa fille Rebeca l'attend à l'aéroport. Elle est nerveuse, les flash-back sur son enfance et sur ses conflits avec les amants de sa mère se succèdent («conflits» est une expression assez faible ici, compte tenu du fait qu'on la voit enfant assassiner un des amants de Becky). Rebeca est d'ailleurs mariée avec un ancien amant de sa mère, Manuel. Leur mariage fonctionne mal et Manuel aura à nouveau une liaison avec Becky, sa belle-mère. Rebeca, quant à elle, tombera enceinte du travesti Femme-Letal, qui imite Becky en play-back. Même si Letal est follement amoureux d'elle, Rebeca ne supporte pas que son mari, Manuel, la trompe et elle le tue avec un revolver. Une enquête policière commence alors, menée par le sérieux juge Dominguez. Rebeca, qui est speakerine à la chaîne de télévision qui appartenait à son mari, confesse son crime en plein téléjournal. On l'envoie en prison, où elle rencontre une femme qui a eu un amant, Hugo, que l'on dit mort, et qui a la même particularité anatomique que Letal, soit un grain de beauté sur le sexe. Le juge Dominguez, qui apprend qu'elle est enceinte, la fait sortir de prison et finit par se dévoiler: il est à la fois Letal et Hugo, il s'est créé ses propres 
indicateurs. Tandis que Dominguez ne croit pas ou ne veut pas croire que Rebeca est l'assassin de Manuel - ce qui finit par arranger Rebeca Becky, très gravement malade, s'accuse du meurtre pour réparer ses torts envers sa fille, pour la couvrir. Mourante, elle demandera gentiment mais fermement à sa fille, comme une mère demande à son enfant, de trouver un autre moyen de régler ses problèmes avec les hommes. Il est clair ici que Letal, Rebeca, Dominguez, Manuel, Becky ne sont pas des cas psychanalytiques, mais bien les personnages d'un film de Pedro Almodovar. La cure n'est pas de rigueur. Il s'agirait simplement de tenter de penser un modèle de la virilité loin du neutre, de l'évidence et du naturel. Ce qui nous intéresse, c'est cette dislocation du masculin comme genre-étalon, comme genre neutre qui est en marche dans le travestissement de Dominguez en Hugo, en Letal et en juge.

Dans Talons aiguilles, le masculin, comme le féminin d'ailleurs, n'est jamais évident, il est toujours le produit d'un déguisement, d'un travestissement.

En refermant ses jambes pour éviter le regard agaçant du mari de Rebeca, Manuel, qui regarde entre les pans de la chemise ouverte, qui tente de voir sous sa jupe, à travers ses bas résilles, et qui le presse de questions sur son sexe, Letal répond d'une voix grave: - «Para ti soy un hombre». Ce dernier dirige alors son regard vers l'entre-jambe de Manuel qui referme son veston, pour dérober à la vue de Letal la crosse de son pistolet. Les deux hommes se sont jaugés du regard. Pourtant, si l'insigne de sa virilité semble se trouver entre les jambes de Manuel, c'est à la crosse du revolver ques'arrêtera la caméra. Et c'est cette mêmecrosse que tentera de cacher Manuel. Un peu plus loin dans le film, nous verrons le regard très surpris du Juge Dominguez qui regarde une photographie de cette soirée sur laquelle on aperçoit encore la crosse du revolver de Manuel, mais cette fois distinctement. Pistolets à remplir de balles, talons aiguilles menaçant de percer; les insignes des sexes viennent nommer un corps qui se dit trop peu hors des signes.

Femme-Letal est un travesti, un «personnificateur féminin» comme le veut l'expression des bars de travestis. Il imite, il bouge les lèvres sur un disque de Becky. Et quand cette dernière lui demande un sein en souvenir, il enlève un de ses faux seins et lui tend. Son travestissement n'aura jamais cessé de nous le faire voir en tant qu'homme travesti en femme, en "femme fatale» comme l'indique son nom, et jamais en tant que femme. Ici aucune confusion n'est possible. Son travestissement garde intacte son identité de mâle. Il est une femelle, il emprunte le genre 
femelle mais ne désire pas changer son sexe de mâle. -«Para ti soy un hombre» a-t-il dit à son rival, le mari de Rebeca. Pas d'opérations, ni de prises d'hormones; pas d'électrolyse, seulement le plaisir ou le désir d'imitation. Un déguisement qui vient l'assurer de son état de mâle, de sa différence $d^{\prime}$ avec les femelles. Perruque, rouge à lèvre, minijupe, gaine; on verra Letal se débarrasser de ses artifices à la scène suivante. Mais il ne complète pas sa transformation, ce qui lui donne un aspect étrange. Il garde un slip et un espèce de casque de bain qui cache ses cheveux. On ne connaît pas sa véritable identité, il ne donne pas son adresse à Rebeca. Plus tard dans le film, on ne connaîtra pas plus sa véritable identité (ce genre de chose existe-t-elle ?), nous le verrons jouer d'autres rôles et c'est tout. Nous aurons tout de même un indice anatomique qui permettra à Rebeca de faire le lien entre Hugo et Letal: il a un grain de beauté sur le sexe. Les yeux, la bouche, le sexe, le corps entier ne suffisaient pas à distinguer Letal d'un autre, il fallait qu'un signe de plus, anecdotique et qui fait sourire, vienne marquer l'insigne de son identité sexuelle.

Moustaches et barbe postiches, lunettes légèrement teintées, veste, pantalon et cravate sombres, chemise à col dur; le juge Dominguez est aussi un travesti, un «personnificateur masculin», comme le veut l'expression qu'utilise Mark Simpson dans son livre Male Impersonators. Il imite, il a les gestes stéréotypés des juges d'instruction de la télévision. La virilité conventionnelle qui accompagne ici la place de pouvoir du juge ne se fait pas sans cravate. La masculinité autorise, elle demande même souvent le port de la cravate; et dans un même temps, cette virilité est nommée en partie par la cravate. Justice des hommes et virilité s'enchevêtrent parodiquement dans Talons aiguilles. Peut-être faut-il rappeler les mots de Freud:

Le fait qu'il faille reconnaîtreà la femme peu de sens de la justice est sans doute lié à la prédominance de l'envie dans sa vie psychique, car l'exigence de justice est une élaboration de l'envie, elle indique à quelle condition on peut la laisser agir. (180)

C'est un avis que partagent certains hommes et certaines femmes aussi d'ailleurs: les femmes sont tricheuses et déloyales de nature. Mais laissons de côté un moment la misogynie ordinaire du sens commun. Que se passe-t-il lorsqu'un juge parfaitement sérieux et viril est un vraifaux juge ? Que se passe-t-il quand la virilité devient une affaire de déguisement, d'artifice? Si les poils pubiens permettent de cacher, selon 
Freud, le manque de la femme (des poils qui auraient fourni aux femmes le modèle qui a permis l'invention du tissage [177-178]), que dissimule donc la barbe bien taillée du juge Dominguez ? Elle cache peu de choses; elle vient marquer le visage d'un code de la virilité, une virilité qui est à lire à travers un ensemble de signes. Ainsi, le tissu du veston et du pantalon qui enveloppe le corps de Dominguez sera de fibres synthétiques. Ce n'est pas l'effet du hasard. Les habits du juge ne montrent pas une «naturalité» de l'état de mâle à qui un sexe masculin suffirait. Col de chemise rigide, port du pistolet, couleur sombre de la veste; tout atteste le sérieux, un sérieux et une masculinité que le travestissement d'homme en homme donne à voir.

Si la place du juge semblait être la seule qui demandait d'être vraie de par ses implications légales particulières, il est amusant de constater que la barbe de Dominguez est fausse et que c'est muni de cet artifice qu'il voit sa maman. Vraie mère, faux fils; dans Talons aiguilles, tout est de l'ordre du vrai-faux. La mère collectionne les articles qui parlent de mère Theresa, de Brigitte Bardot et de Becky, qui, elle, est une pure invention du scénario du film. On est dans la vérité et le mensonge. Dans la réalité, le père de l'acteur qui joue le travesti est le célèbre torero «Dominguin». Almodovar joue avec cette virilité vraie et réelle et la donne au fils acteur qui dans le film s'appelle juge «Dominguez». Miguel Bosé, l'acteur, joue le rôle d'un homme qui a un nom viril et semblable à celui de son père réel. Le faux et le vrai se côtoient et viennent se contaminer l'un l'autre.

Lorsque, vers la fin du film, Rebeca entre dans la loge de Letal, elle trouve à son grand étonnement des verres de contact et une barbe postiche. Mais ce moment de dévoilement de la vérité est étrange; HugoLetal-juge Dominguez apparaît à demi transformé. Un peu après cette scène, le travesti, déguisé en Hugo, tentera de lui expliquer qui il est. On a alors l'impression que Hugo, c'est le vrai personnage. Ni trop masculin, ni trop féminin. Quelqu'un de normal. Un homme en jean et en t-shirt. Mais Hugo est déjà un autre, junkie indicateur, déjà mort selon certains. Petit ami de l'une, petit ami de l'autre, ego-Hugo n'est ni la clef de l'énigme ni l'individu original sur le corps duquel se fait le travestissement en femme et en homme. Il dira, pour expliquer ses déguisements, qu'il s'est créé ses propres indicateurs. On pourrait ajouter: comme le travesti indique son genre.

Le film tout entier nous invite à mettre ensemble les indices. En citant, entre autres, un film hollywoodien de 1959, Imitation of life, qui était déjà 
lui-même une reprise d'un film plus ancien, Almodovar marque son désir de n'être jamais dans une authenticité qui n'existe pas de toute façon. - «Cesse de jouer maman !», implore Rebeca. On entendait déjà cette réplique dans Imitation of life, mais il demeure impossible de sortir de l'imitation ${ }^{1}$. L'authentique fait déjà partie d'un système de représentation, on n'en sort pas. Dans ce jeu des apparences, le dernier rempart de l'authenticité, de l'adéquation naturelle du corps à son sexe, de $l^{\prime}$ «organicité» de son être, soit l'homme viril, se dissout lui aussi. Et la virilité ne s'élabore plus qu'à partir du travestissement $d^{\prime}$ 'un corps qui ne parle pas de lui-même. Nous restons toujours ici dans l'imitation. Comme le dit Butler:

To claim that all gender is like drag, or is drag, is to suggest that «imitation» is at the heart of the heterosexual project and its gender binarisms, that drag is not a secondary imitation that presupposes a prior and original gender, but that hegemonic heterosexuality is itself a constant and repeated effort to imitate its own idealizations.

L'homme hétérosexuel viril ou le «macho» comme le veut l'expression, même s'il demeure dominant comme modèle, $n^{\prime}$ a pas toujours bonne presse. On le dit coupable de tous les maux. On lui reproche de se faire sur la haine de l'homosexuel mâle, sur la haine de la femme. Ainsi, le nouvel homme ou l'homme rose, sous-entendu blanc, occidental et de préférence hétérosexuel, ne doit pas marquer de façon importante son identité sexuelle. On ne doit pas faire trop de cas de cette identité, puisqu'elle participe de l'évidence. Ses vêtements, à défaut d'être neutres, se veulent discrets. Il cherche à gommer les marques du masculin, il préfère avoir une attitude que l'on dit naturelle, être à l'aise, confortable et bien dans sa peau. Pourtant, si le mâle viril a souvent endossé à lui seul plusieurs millénaires d'oppression des femmes, peutêtre est-il aujourd'hui possible de l'envisager, pour un moment, comme subversion d'un masculin qui se veut neutre et qui est, en définitive, aussi hégémonique qu'un masculin qui veut atteindre la virilité. Ce mâle viril se démènerait comme la femme, comme le ou la travesti-e, avec un travestissement qui marquele genre, avec la difficile différence sexuelle. Bien entendu, il n'existe jamais une attitude qui nous assure d'être pour de bon hors de tous soupçons; il est clair que les discours sur la virilité sont le plus souvent particulièrement réactionnaires. 
Dans Matador, un autre film de Pedro Almodovar, l'identité sexuelle est aussi traduite, marqueé, travestie. Dans une des scènes du film, on voit une femme très féminine, très habillée (pour le travail d'ailleurs, elle est avocate), entrer dans les toilettes des hommes d'un cinéma. Un homme la suit. Elle lui demande pourquoi il la suit. Il lui dit: $-«$ Tu es dans les toilettes des hommes, tu n'as pas vu le signe ?» Et elle de répondre «avec mépris», comme le dit le scénario: -«Ne te fie pas aux apparences». La scène est filmée entièrement dans le miroir. Ici, qui est masculin? qui est féminin ? Les apparences sont trompeuses, les signes difficiles à interpréter. Le sexe et le genre surtout ne se donnent pas en une fois. Une stratégie aura voulu donner à penser la masculinité comme étant évidente et neutre. Le féminin dans cette stratégie vient toujours se donner à lire dans son écart par rapport au neutre, au masculin neutre. Que l'on pense à la difficile question de la féminisation des noms. L'accord réglé par le masculin, dans plusieurs langues, participe d'une stratégie qui vise à établir ou à maintenir un sexe inclusif de l'autre. Comme l'écrit Irigaray dans Parler n'est jamais neutre:

Une loi, perpétuellement méconnue, prescrit toutes réalisations de langage(s), toute production de discours, toute constitution de langue, selon les nécessités d'une perspective, $\mathrm{d}^{\prime} u n$ point de vue, d'une économie: celles de l'homme, supposé représenter le genre humain. (281)

Les académiciens français qui prescrivent le neutre masculin, même s'ils sont mâles, ne sont pas neutres ou impartiaux. Que l'on pense aux théories freudiennes sur l'identité sexuelle et à l'obsession de Freud pour un sexe mâle vu comme seule articulation de la différence des sexes. Nous sommes pris dans la différence, dans une différence difficile que l'envie ne cesse de menacer. Il n'en demeure pas moins que l'homme ne peut jamais être neutre; sa masculinité se manifeste toujours, elle aussi, dans son écart par rapport au neutre, par rapport à ce qui n'est pas sexué, à ce qui n'est ni l'un ni l'autre. Le dispositif de Talons aiguilles met en scène, souligne l'impossibilité d'un sexe biologique qui donnerait une fois pour toutes le bon genre. Le personnage de Letal-Dominguez-Hugo, en femme ou en homme, montre à quel point on ne peut jamais sortir du travestissement, d'une opération qui vise à marquer un genre que le biologique ne peut marquer entièrement et de façon définitive.

- -Ne te fie pas aux apparences». 


\section{Références}

Boehm, Felix. «Le complexe de féminité chez l'homme». Nouvelle revue de psychanalyse, No. 7 (printemps 1973), p. 277-300.

Butler, Judith. Bodies that Matter: On the Discursive Limits of Sex. New York: Routledge, 1993.

Freud, Sigmund. Nouvelles conférences d'introduction à la psychanalyse. Paris: Gallimard, 1984.

Irigaray, Luce. Parlern'est jamais neutre. Paris: Gallimard, 1985.

Simpson, Mark. Male Impersonators: Men Performing Masculinity.

Londres et New York: Routledge, 1994.

Smith, Paul Julian. Desire Unlimited: The Cinema of Pedro Almodovar. Londres: Verso, 1994.

\section{Notes}

1 Voir Paul Julian Smith, Desire Unlimited: The Cinema of Pedro Almodovar (127-130).

Ce texte a été présenté pour la première fois dans le cadre du Sixième colloque annuel des étudiantes et étudiants des deuxième et troisième cycles de l'Association canadienne de littérature comparée («Corps/Corpus. The Body of Literature/Literature of the Body»), tenu au Département de litterature comparée de l'Université de Montréal les 31 mars et 1er avril 1995 et organisé par Marie Lessard et Craig Ireland. 